\title{
An open label trial of folate receptor-targeted intraoperative molecular imaging to localize pulmonary squamous cell carcinomas
}

\author{
Jarrod D. Predina ${ }^{1,2}$, Andrew D. Newton ${ }^{1,3}$, Leilei Xia $^{1,4}$, Christopher Corbett ${ }^{1,3}$, \\ Courtney Connolly ${ }^{1,2}$, Michael Shin ${ }^{1,2}$, Lydia Frezel Sulyok ${ }^{1,2}$, Leslie Litzky ${ }^{5}$, \\ Charuhas Deshpande ${ }^{5}$, Shuming Nie ${ }^{6}$, Sumith A. Kularatne ${ }^{7}$, Philip S. Low ${ }^{7}$ and \\ Sunil Singhal ${ }^{1,2}$ \\ ${ }^{1}$ Center for Precision Surgery, Perelman School of Medicine at The University of Pennsylvania, Philadelphia, PA, USA \\ ${ }^{2}$ Division of Thoracic Surgery, Department of Surgery, Perelman School of Medicine at The University of Pennsylvania, \\ Philadelphia, PA, USA \\ ${ }^{3}$ Department of Surgery, Perelman School of Medicine at The University of Pennsylvania, Philadelphia, PA, USA \\ ${ }^{4}$ Division of Urology, Department of Surgery, Perelman School of Medicine at The University of Pennsylvania, Philadelphia, \\ PA, USA \\ ${ }^{5}$ Pathology and Laboratory Medicine at The Hospital of The University of Pennsylvania, Philadelphia, PA, USA \\ ${ }^{6}$ Department Biomedical Engineering and Winship Cancer Institute, Emory University, Atlanta, GA, USA \\ ${ }^{7}$ Department of Chemistry, and Purdue Institute for Drug Discovery, Purdue University, West Lafayette, IN, USA \\ Correspondence to: Sunil Singhal, email: Sunil.singhal@uphs.upenn.edu \\ Keywords: squamous cell carcinoma; surgery; molecular imaging; folate receptor \\ Received: November 21, $2017 \quad$ Accepted: January 09, $2018 \quad$ Published: February 05, 2018 \\ Copyright: Predina et al. This is an open-access article distributed under the terms of the Creative Commons Attribution License \\ 3.0 (CC BY 3.0), which permits unrestricted use, distribution, and reproduction in any medium, provided the original author and \\ source are credited.
}

\section{ABSTRACT}

Background: Clinical applicability of folate receptor-targeted intraoperative molecular imaging (FR-IMI) has been established for surgically resectable pulmonary adenocarcinoma. A role for FR-IMI in other lung cancer histologies has not been studied. In this study, we evaluate feasibility of FR-IMI in patients undergoing pulmonary resection for squamous cell carcinomas (SCCs).

Methods: In a human clinical trial (NCT02602119), twelve subjects with pulmonary SCCs underwent FR-IMI with a near-infrared contrast agent that targets the folate receptor- $\alpha(F R \alpha)$, OTL38. Near-infrared signal from tumors and benign lung was quantified to calculate tumor-to-background ratios (TBR). Folate receptoralpha expression was characterized, and histopathologic correlative analyses were performed to evaluate patterns of OTL38 accumulation. An exploratory analysis was performed to determine patient and histopathologic variables that predict tumor fluorescence.

Results: 9 of 13 SCCs (in 9 of 12 of subjects) displayed intraoperative fluorescence upon NIR evaluation (median TBR, 3.9). OTL38 accumulated within SCCs in a FRadependent manner. FR-IMI was reliable in localizing nodules as small as $1.1 \mathrm{~cm}$, and prevented conversion to thoracotomy for nodule localization in three subjects. Upon evaluation of patient and histopathologic variables, in situ fluorescence was associated with distance from the pleural surface, and was independent of alternative variables including tumor size and metabolic activity.

Conclusions: This work demonstrates that FR-IMI is potentially feasible in $70 \%$ of SCC patients, and that molecular imaging can improve localization during 


\section{minimally invasive pulmonary resection. These findings complement previous data demonstrating that $\mathbf{9 8} \%$ of pulmonary adenocarcinomas are localized during FR-IMI and suggest broad applicability for NSCLC patients undergoing resection.}

\section{INTRODUCTION}

Each year, nearly 200,000 Americans are diagnosed with non-small cell lung cancer (NSCLC) [1]. Surgical resection provides the best opportunity for long-term survivorship for NSCLC patients, and nearly 80,000 patients undergo pulmonary resection in the United States annually [1]. Although the use of minimally invasive pulmonary resection (video-assisted thoracic surgery or robotic surgery) for NSCLC is common, these approaches limit a surgeon's ability to visually inspect and palpate the entire lung [2]. Consequential incomplete intraoperative information during minimally invasive pulmonary resection can make localization and adequate resection of tumors challenging.

Intraoperative molecular imaging (IMI), also known as fluorescence guided surgery, is a rapidly evolving technique that has been demonstrated to improve the surgeon's ability to accurately identify malignant nodules during oncologic resection [3-10]. This approach incorporates the systemic delivery of optical contrast agents which preferentially accumulate in malignant tissues, and allows for real-time fluorescent imaging using calibrated camera systems. IMI has been effectively utilized for a variety of malignancies including thoracic malignancies [11-14], central nervous system tumors [15], head and neck cancers [8], ovarian carcinomas [5], and thymoma [16].

Over the last decade our group has initiated several human clinical trials involving folate receptor-targeted intraoperative molecular imaging (FR-IMI) for the most common NSCLC histology, pulmonary adenocarcinomas $[3,11,13,17-19]$. Pulmonary adenocarcinomas were chosen as an initial disease histology given a high level of FR $\alpha$ expression which approaches $85 \%[13,20,21]$. In a Phase I trial, we found FR-IMI with OTL38 to be both safe and feasible. After optimizing imaging parameters, we appreciated that more than $90 \%$ of pulmonary adenocarcinomas accumulated OTL38 and displayed fluorescence in situ or ex vivo [13]. In a follow-up study involving 50 subjects, the addition of FR-IMI to standard of care intraoperative assessment allowed for detection of occult subcentimeter nodules in $12 \%$ of subjects [3]. Together, these intraoperative findings impacted operative planning, tumor staging, and postoperative adjuvant therapy treatment.

Although initial trials with FR-IMI have been successful for localization of pulmonary adenocarcinomas, utility for other NSCLC histologies remains undefined. Pulmonary squamous cell carcinomas (SCCs) account for approximately $30 \%$ of all lung cancers and 35\% of NSCLCs, thus exploration of IMI with OTL38 in
SCCs has the potential for high clinical impact [22]. Unfortunately, there is currently a paucity of information describing FR $\alpha$ expression patterns in SCCs $[20,21]$. Further, the few reports describing FR $\alpha$ expression provide conflicting data, with FR $\alpha$ expression levels ranging from $10 \%$ to $60 \%$ [20, 21]. This limited information has impeded progress of folate-receptor targeted molecular imaging for SCCs.

As our group prepares to move FR-IMI with OTL38 to Phase II evaluations, we sought to better understand the utility of FR-IMI in patients with SCC. In this report, we evaluate FR-IMI with OTL38 in 12 subjects undergoing minimally invasive pulmonary resection for SCCs. In this study, our primary goals were to determine if OTL38 reliably accumulates within SCC and generates tumor fluorescence. Our secondary goals were to characterize folate receptor expression within SCC's and determine factors which contribute to tumor fluorescence during FR-IMI.

\section{RESULTS}

\section{Subject and drug delivery data}

Between August 2015 and February 2017, 12 subjects with a histologically confirmed diagnosis of pulmonary SCC underwent VATS resection with FR-IMI with OTL38. Seven included subjects were male and 5 were female. The median age of subjects at resection was 70 years (IQR, 6877 years). Except for Subject 5, all subjects had a solitary pulmonary nodule by preoperative imaging. Subject 5 had presented with two synchronous nodules: one in the left upper lobe and one in the left lower lobe. Preoperative $\mathrm{CT}$ and PET were obtained in all subjects and the median tumor size of SCCs included in analysis was $1.7 \mathrm{~cm}$ (IQR, $1.5-4.0 \mathrm{~cm}$ ) with median SUV of 4.8 (IQR, 3.5-8.9). A full summary of patient characteristics is provided in Table 1.

Subjects received a median of $2.07 \mathrm{mg}$ (IQR, 1.77$2.58 \mathrm{mg}$ ) of OTL38 4.4 hours (IQR, 3.4-5.1 hours) prior to resection and imaging (Table 2). Each subject was administered the full amount of study drug. No adverse events were noted during 30 days of follow-up.

\section{SCCs displaying fluorescence during FR-IMI}

In situ molecular imaging using the Iridium's thoracoscopic configuration identified tumor-specific fluorescence signal through the pleural surface in 7 $(58.3 \%)$ of 12 subjects (Figure 1a-1d; Supplementary Video 1), with 7 of 13 (53.8\%) nodules being localized by NIR imaging. Upon thoracoscopic NIR evaluation, the median tumor fluorescence intensity was noted to be 91.0 
Table 1: Subject characteristics

\begin{tabular}{|c|c|c|c|c|c|c|c|c|c|}
\hline ID & $\begin{array}{c}\text { Age } \\
\text { (years) }\end{array}$ & Gender & $\begin{array}{c}\text { Tumor } \\
\text { Location }\end{array}$ & Size $(\mathrm{cm})$ & $\begin{array}{c}\text { Depth } \\
\text { (cm) }\end{array}$ & SUV & In situ TBR & $\begin{array}{c}\text { Ex vivo } \\
\text { TBR }\end{array}$ & Impact of IMI \\
\hline 1 & 70 & $\mathrm{~F}$ & RLL & 5.2 & 2.0 & 13.1 & 1.3 & 4.3 & \\
\hline 2 & 87 & $\mathrm{~F}$ & RUL & 1.0 & 0.1 & avid $^{*}$ & 1.6 & 1.8 & \\
\hline 3 & 77 & $\mathrm{~F}$ & LUL & 1.7 & 0.0 & 8.7 & 3.7 & 4.2 & \\
\hline $4^{* *}$ & 60 & $\mathrm{~F}$ & $\begin{array}{l}\text { LUL } \\
\text { LLL }\end{array}$ & $\begin{array}{l}0.9 \\
1.2\end{array}$ & $\begin{array}{l}0.0 \\
0.4\end{array}$ & $\begin{array}{l}3.0 \\
3.5\end{array}$ & $\begin{array}{l}1.0 \\
1.2\end{array}$ & $\begin{array}{l}1.9 \\
1.9\end{array}$ & \\
\hline 5 & 69 & M & RLL & 1.5 & 0.5 & 8.9 & 2.2 & 5.6 & $\begin{array}{c}\text { Improved } \\
\text { minimally invasive } \\
\text { localization }\end{array}$ \\
\hline 6 & 77 & M & RLL & 4.6 & 0.2 & 8 & 2.4 & 2.6 & \\
\hline 7 & 70 & M & RUL & 4.0 & 0.0 & 18.3 & 3.8 & 3.9 & \\
\hline 8 & 67 & M & RLL & 2.8 & 0.0 & 5.1 & 3.0 & 3.8 & \\
\hline 9 & 78 & M & RUL & 1.1 & 1.2 & 3.1 & 2.2 & 3.5 & $\begin{array}{c}\text { Improved } \\
\text { minimally invasive } \\
\text { localization }\end{array}$ \\
\hline 10 & 72 & M & RUL & 3.0 & 4.0 & 4.5 & 1.0 & 3.9 & \\
\hline 11 & 66 & M & RUL & 4.5 & 2.5 & 2.5 & 1.3 & 1.4 & \\
\hline 12 & 70 & $\mathrm{~F}$ & RUL & 1.7 & 0.9 & 3.8 & 2.1 & 2.4 & $\begin{array}{c}\text { Improved } \\
\text { minimally invasive } \\
\text { localization }\end{array}$ \\
\hline
\end{tabular}

M-Male; F-Female; RUL-Right Upper Lobe; RML-Right Middle Low; RLL-Right Lower Lobe; LUL-Left Upper Lobe; LLL-Left Lower Lobe.

* unable to quantify as records from outside our health system.

${ }^{* *}$ Subject with two nodules identified preoperatively. The larger was PET avid, with suggesting SCC. The second nodule was not biopsied preoperatively and was not PET avid; however, proved to be SCC on final histopathologic analysis.

AU (IQR, 83-105 AU) (Figure 1e), which was higher than within surrounding benign pulmonary parenchyma which measured 28.5 AU (IQR, 23-45 AU) (Figure 1f); $<<0.001$ (Figure 1g). The median in situ TBR of nodules displaying in situ fluorescence was 2.4 (IQR, 2.2-3.7). The median size of the $7 \mathrm{in}$ situ fluorescent tumors was $1.7 \mathrm{~cm}$ (range, $1.1-4.6 \mathrm{~cm}$ ). Based on preoperative imaging, each of these tumors were sub-pleural and located within $1.2 \mathrm{~cm}$ of the lung surface.

After resection and direct tumor exposure during ex vivo tumor bisection, two SCCs which were nonfluorescent by thoracoscopic assessment displayed obvious ex vivo fluorescence upon tumor bisection (Subject 1 and Subject 10) (Figure 2a-2f; Supplementary Video 2). All (7 of 7) SCCs exhibiting in situ signal also displayed fluorescence upon exoscopic FR-IMI. In total, 9 of 13 (sensitivity 69.2\%; false negative rate of $31.8 \%$ ) evaluated SCCs evaluated (in 9 of 12 subjects) displayed strong signal during FR-IMI. We noted uniform tumorassociated fluorescence throughout the tumor surface with a clear demarcation noted between the tumor margin and benign surrounding lung parenchyma (Figure 2f). Median fluorescence of these 9 SCCs was nearly 4-fold higher than signal of surrounding benign lung (103 vs 29 AU; $\mathrm{p}<0.001$ ) (Figure 2g-2i). The median TBR of those SCCs displaying ex vivo fluorescence upon bisection was 3.9 (IQR, 3.5-4.2); which was higher than TBRs observed during in situ fluorescent evaluation; $p=0.02$. Of note, lesions displaying only signal upon ex vivo evaluation were similar in size to those displaying in situ (median $4.1 \mathrm{~cm}$ vs $1.7 \mathrm{~cm} ; \mathrm{p}=0.18$ ); however, were deeper than those also displaying in situ signal (median $3.0 \mathrm{~cm}$ vs. $0.2 \mathrm{~cm} ; \mathrm{p}<0.01)$.

\section{Folate receptor-targeted OTL38 accumulation within SCCs}

Of those SCCs demonstrating fluorescence upon FR-IMI, each demonstrated FR $\alpha$ expression $(1+, 2+$ or $3+$ ) upon immunostaining (representative examples of $0,1+, 2+$ and $3+$ staining provided in Figure 3$)$. Three (33.3\%) FR $\alpha$-expressing tumors were found to have 


\begin{tabular}{|c|c|c|c|c|}
\hline ID & Weight (kg) & OTL38 given (mg) & Adverse Event ${ }^{*}$ & $\begin{array}{l}\text { Time from Infusion to } \\
\text { Imaging (hours) }\end{array}$ \\
\hline 1 & 61.8 & 1.55 & & 2.97 \\
\hline 2 & 63.5 & 1.59 & & 3.38 \\
\hline 3 & 61.7 & 1.54 & & 5.73 \\
\hline 4 & 79.8 & 2.00 & & 4.40 \\
\hline 5 & 134.3 & 3.36 & & 4.72 \\
\hline 6 & 102.1 & 2.55 & & 6.5 \\
\hline 7 & 107 & 2.68 & & 6.45 \\
\hline 8 & 73.5 & 1.84 & & 3.68 \\
\hline 9 & 82.8 & 2.07 & & 5.05 \\
\hline 10 & 103 & 2.58 & & 3.15 \\
\hline 11 & 70.8 & 1.77 & & 4.18 \\
\hline 12 & 88.5 & 2.21 & & 2.98 \\
\hline
\end{tabular}

*Adverse Events were graded using the Common Terminology Criteria for Adverse Events (Version 4.03) [33].

predominantly $1+$ staining, $4(44.4 \%)$ had predominantly $2+$ staining patterns, and $2(22.2 \%)$ had predominantly $3+$ staining. In each FR $\alpha$-positive specimen, heterogenous expression patterns were appreciated with each specimen displaying areas of $0,1+, 2+$ and $3+$ staining. Of note, FR $\alpha$-expression was absent in the three non-fluorescent SCCs submitted for histopathologic review.

To better characterize patterns of OTL38 accumulation within SCC and confirm accumulation in FR $\alpha$-expressing tumors areas, all fluorescent SCC underwent microscopic tumor mapping using a NIR scanning system and molecular correlative analysis using a combination of H\&E staining and FR $\alpha$ immunohistochemistry (representative analysis provided in Figure 4). After noting tumor margins by of $H \& E$ and IHC, quantitative fluorescent analysis confirmed elevated mean fluorescence intensities within tumors as compared to normal benign pulmonary parenchyma (versus $98.1 \mathrm{AU}$ vs 20.3; $<<0.001$ ) (Figure 4). Within tumors, we noted significant increases in fluorescent intensity in those areas with FR $\alpha$-overexpression ( $2+$ or $3+$ staining) versus those areas with lower expression patterns (140.6 AU vs 57.2 $\mathrm{AU} ; \mathrm{p}<0.001)$.

\section{FR-IMI improves localization of T1a squamous cell carcinomas}

After confirming FR $\alpha$-dependent accumulation of OTL38 within SCC, we reviewed how FR-IMI impacted clinical care. In 3 of 12 subjects, utilization of FR-IMI impacted surgical care by allowing for intraoperative localization of T1a lesions (measuring less than $2.0 \mathrm{~cm}$ ).
For example, Subject 9 presented with a ${ }^{18}$ FDG-avid, $1.1 \mathrm{~cm}$ right upper lobe nodule (Figure 5a, 5b). This patient was taken to the operating room for a VATS wedge resection. During standard thoracoscopic localization (white light and finger palpation), the nodule could not be localized through the pleural surface (Figure $5 \mathrm{c}$ ). However, after implementation of in situ NIR molecular imaging, the nodule was readily identified (Figure 5d). Using the real-time fluorescent feedback, the lesion was successfully resected and adequate margins were confirmed by exoscopic fluorescent evaluation. Fluorescent results were confirmed by both frozen section and final pathologic evaluation. Without the addition of FR-IMI, the operating surgeon would have otherwise converted from VATS to thoracotomy to accomplish localization and resection. Similarly, in Subject 5 and Subject 12 the addition of FRIMI allowed for the resection of T1a nodules to continue in a minimally invasive fashion.

\section{Histopathologic and clinical variables predicting in-situ fluorescence of SSCs during FR-IMI}

To maximize utility of FR-IMI for localization, in situ intraoperative fluorescence is paramount. To determine which patient and tumor variables predict in situ signal upon FR-IMI; several clinical, radiographic and histopathologic variables were assessed: depth from pleural surface on preoperative $\mathrm{CT}$, time from infusion to imaging, preoperative SUV, tumor location, SCC size, amount of drug given, subject age and final subject gender (Table 3). Of the 9 tumors which accumulated OTL38, we determined that only depth from pleural surface impacted 
in situ fluorescence, with deeper nodules associated with less fluorescence $(p=0.005)$. In fact, among the 12 subjects analyzed, only those with SCCs within $1.2 \mathrm{~cm}$ of the pleural surface displayed identifiable signal during in situ FR-IMI. Other trends predicting increased in situ TBR included longer time to imaging, however, this did not reach significance $(\mathrm{p}=0.06)$. No other examined variable predicted in situ tumor fluorescence.
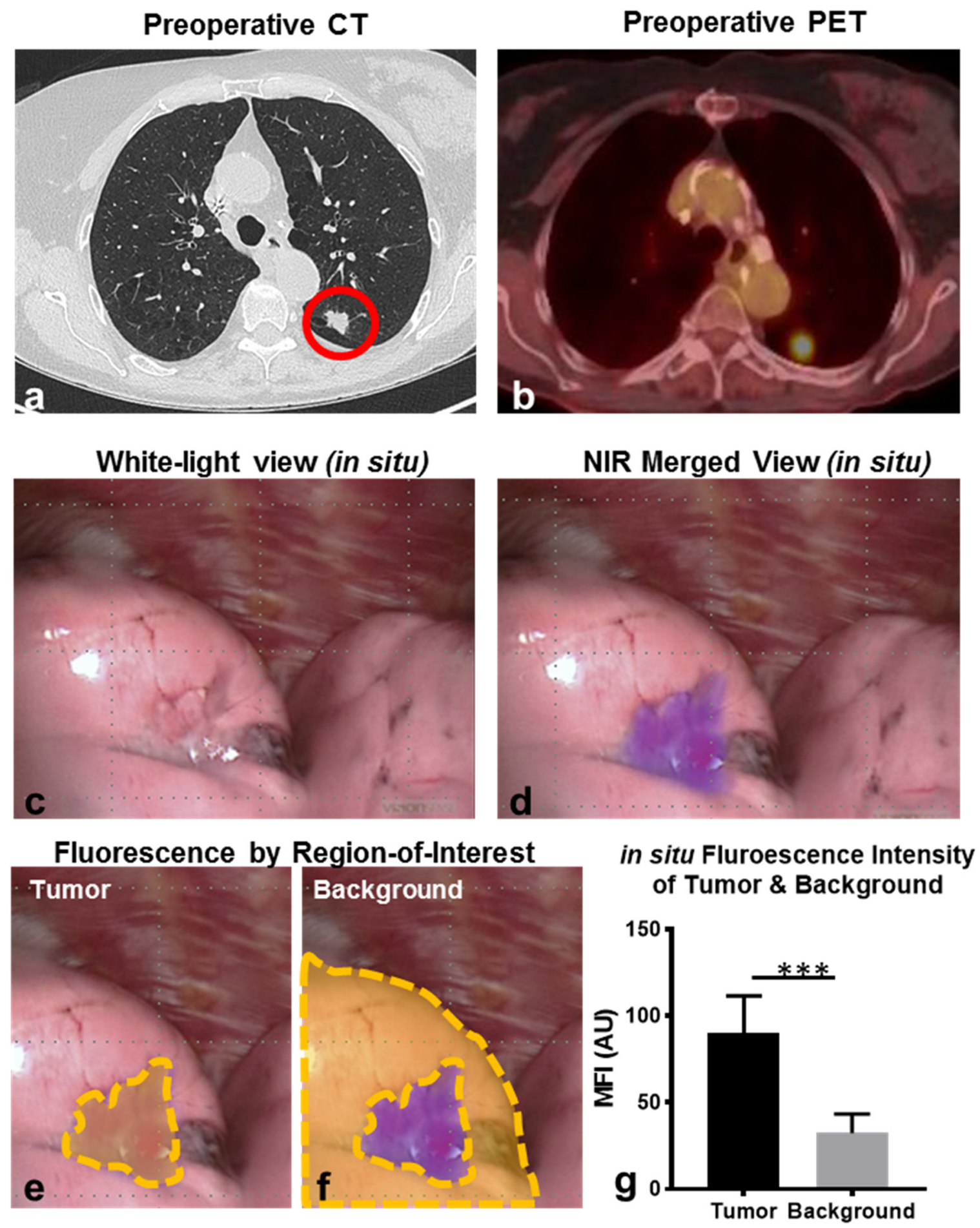

Figure 1: Pulmonary SCCs display in situ fluorescence during FR-IMI with OTL38. Representative example: Subject 3 presented with a $1.7 \mathrm{~cm}$ left upper lobe nodule by preoperative CT (a). Preoperative PET displayed an SUV of 8.7 (b). During FR-IMI the nodule was localized during standard thoracoscopic views (c), and displayed strong NIR signal during fluorescent imaging (d). Median fluorescent intensity (MFI) was determined for ROIs corresponding to tumor (e) and benign lung (background) (f). MFIs for ROIs were compared for SCCs $(\mathrm{n}=7)$ displaying in situ signal (g). Red circle-pulmonary SCC; yellow gates-ROIs measured by fluorescent analysis; ${ }^{* * *} \mathrm{p}<0.001$. 


\section{DISCUSSION}

More than 80,000 patients undergo resection for NCSLC in the United States each year [1]. Localization of pulmonary nodules, particularly during minimally invasive pulmonary resection, can be challenging. Our group has previously demonstrated that FR-IMI with OTL38 can be a safe and reliable intraoperative adjunct to improve localization of pulmonary adenocarcinomas. In this study, we expand upon our previous experiences, and demonstrate FR-IMI with OTL38 can also be a useful surgical adjunct for patients undergoing pulmonary resection of pulmonary SCCs. We demonstrate that OTL38 accumulates in nearly $70 \%$ of SCCs by exhibiting FR $\alpha$-targeted mechanisms. Reproducible fluorescent signal during FR-IMI improved intraoperative localization of small, subpleural nodules during minimally invasive resection.

OTL38 was developed in efforts to incorporate advantages of several alternative imaging agents currently under Phase I/II evaluation. More specifically, OTL38 incorporates highly specific FR $\alpha$-targeted binding as previously observed with EC17 (folate-FITC) [18]. Unlike EC17, folate is linked to S0456 (a commercially available NIR dye) in OTL38 [23]. NIR imaging agents offer several advantages over visual-range agents including minimal autofluorescence and improved depth of tissue penetration [17]. Given an abundance of data describing FR $\alpha$ expression within $70-80 \%$ of pulmonary adenocarcinomas [24], we chose to initiate first-in-human studies using this histology as a study model. In these early trials involving more than 70 patients; FR-IMI with OTL38 was noted to be safe, highly sensitive in localizing small pulmonary adenocarcinomas (as small as $2-3 \mathrm{~mm}$ ), and capable identifying occult malignancy in $12 \%$ of subjects enrolled $[3,13]$.

In light of high reproducibility and excellent sensitivity of FR-IMI within pulmonary adenocarcinomas, we chose to explore FR-IMI with OTL38 in the second most common NSCLC histology, SCCs. SCCs account for nearly $30 \%$ of all lung cancers, and nearly $35 \%$ of all NSCLC histologies. Unlike pulmonary adenocarcinomas, previous data describing FR $\alpha$ expression patterns in SCCs was unclear and ranged from $10 \%$ to nearly $60 \%[20,21]$. In addition to inconsistent expression values, previous reports have been limited due to a small sample size, sampling error introduced by microarray analysis, and poor generalizability to surgical patients. Because of these limitations we sought to execute a feasibility study aimed at exploring FR-IMI in patients with resectable pulmonary SCC.

In this study, we were encouraged to that find 9 of 12 subjects successfully underwent FR-IMI with OTL38. In addition to reproducibility, we noted that FR-IMI provided subcentimeter sensitivity and an effective adjunct to minimally invasive pulmonary resection. Upon fluorescent and correlative histopathologic assessment, we confirm that OTL38 accumulation in SCCs was indeed targeted to FR $\alpha$ expressing areas within tumor. In total, $69 \%$ of SCCs
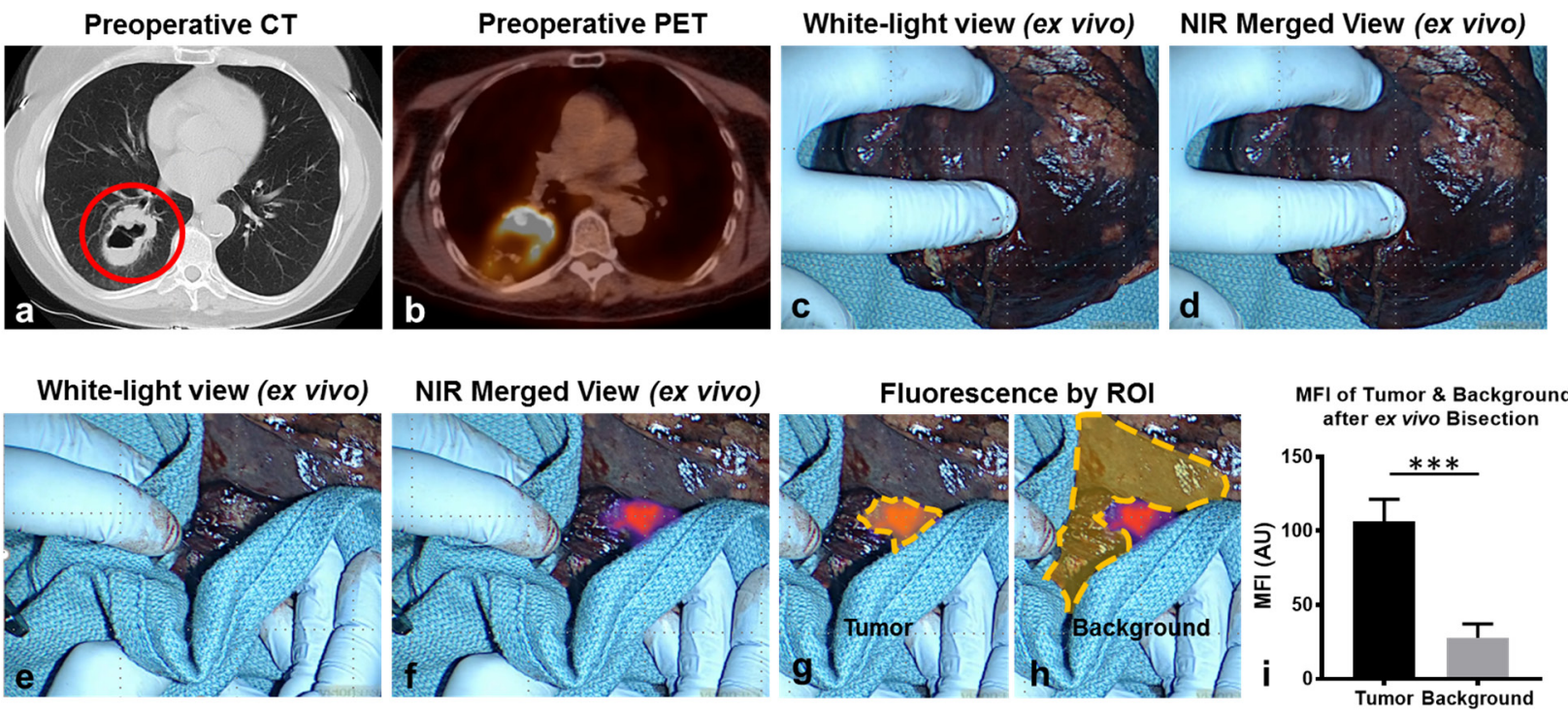

Figure 2: SCCs display strong fluorescence upon ex vivo tumor bisection. Representative example: Subject 1 presented with a $5.2 \mathrm{~cm}$ right lower lobe nodule (a) that displayed an SUV of 13.1 (b). During in situ and ex vivo analysis, no parenchymal changes were appreciated during white light evaluation (c) nor during NIR molecular imaging (d). Upon ex vivo tumor bisection, the tumor was visualized both by white light inspection (e) and by NIR molecular imaging (f). Fluorescent intensity was quantified for ROIs corresponding to the tumor (g) and benign pulmonary parenchyma (background) (h). Mean fluorescent intensities were compared for SCCs ( $\mathrm{n}=9$ ) displaying ex vivo fluorescence during back-table inspection (i). Red circle-pulmonary SCC; yellow gates-ROIs measured by fluorescent analysis; ${ }^{* * *} \mathrm{p}<0.001$. 
displayed FR $\alpha$ expression. We point out that higher rates of FR $\alpha$ expression in our cohort as compared to previous studies may be explained by several factors, perhaps most notably involves our histologic evaluation approach which utilized whole sections as compared to tissue microarrays in previous studies. Furthermore, we observed significant intratumoral heterogeneity in FR $\alpha$ expression as compared to our prior experiences involving pulmonary adenocarcinomas. Nevertheless, these results support our previous findings insomuch tumors display macroscopic fluorescence when as little as $10 \%$ cells display the FR $\alpha$ $[3,13,25]$. These results provide an encouraging platform for future studies that can better clarify these trends in pulmonary squamous cell carcinomas.

Similar to previous studies involving FR-IMI and alternative NIR optical contrast agents, we found significant limitations of with tumors deeper than $1.2 \mathrm{~cm}$. This mirrors our experiences with pulmonary adenocarcinomas where in situ nodule localization was ineffective for lesions deeper than $2.0 \mathrm{~cm}[3,13]$. At this time a number of additional techniques, such as photoacoustic imaging and NIR-endoscopy, are in development. These approaches have proven successful for metastatic melanoma [26] and head and neck malignancies [27], respectively.

Despite these limitations, molecular imaging offers several advantages over alternative localization approaches currently available. First, from a purely logistical perspective, molecular imaging requires no additional procedures which contrasts with CT-guided percutaneous wire localization, fluoroscopy, fiducial marking, and tumor tattooing [28]. In comparison, during
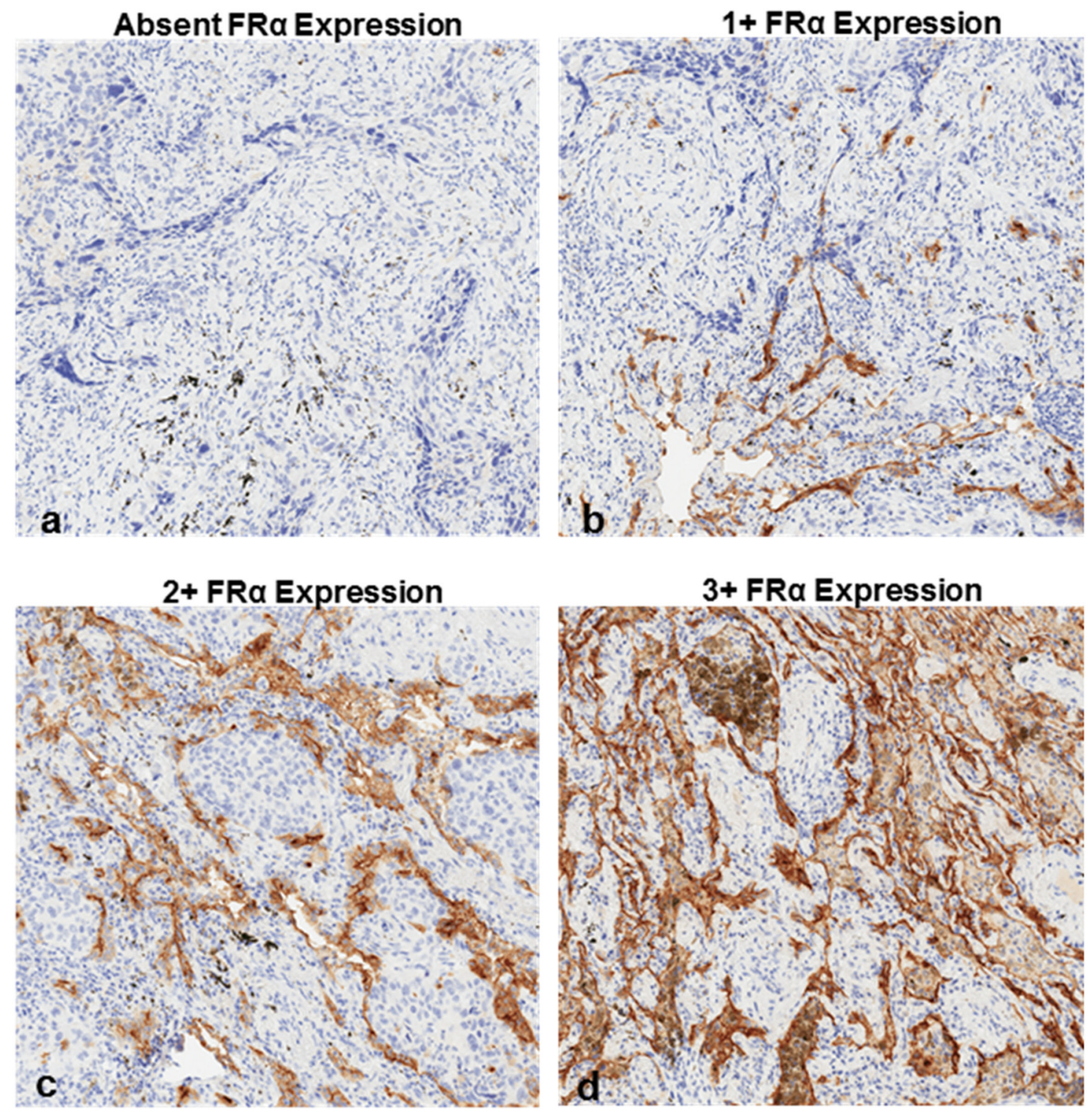

Figure 3: FR $\alpha$ expression patterns in SCCs as determined by immunostaining. (a) absent FR $\alpha$ expression, (b) $1+$ FR $\alpha$ expression, (c) 2+ FR $\alpha$ expression and (d) 3+FR $\alpha$ expression. 2+ and 3+ staining patterns were considered overexpression. 
FR-IMI OTL38 is delivered just hours prior to resection in the preoperative holding area. Secondly, FR-IMI for lung cancer has demonstrated an excellent safety profile with only grade I toxicities noted $[3,13]$. We note a good safety profile in this trial, with no adverse events being observed in this study cohort. This provides important morbidity advantages over previously mentioned localization techniques [29]. Finally, systemic drug delivery involved in IMI provides an opportunity for detection synchronous disease and lymph nodes evaluation as previously noted in our experiences with FR-IMI in pulmonary adenocarcinomas and other groups investigating antibodybased approaches [3, 30].

Despite limitations, we are optimistic about the role of FR-IMI for patients with pulmonary SCCs and view these results as a template for additional studies which can help better define how FR-IMI can improve upon standard treatment approaches for NSCLC patients. More specifically, we are currently exploring FR-IMI with OTL38 as a tool to improve localization of ill-defined pulmonary ground-glass opacities (NCT02769156). In addition to this registration trial, we are embarking on a multi-center Phase II trial (NCT02872701) involving FR-
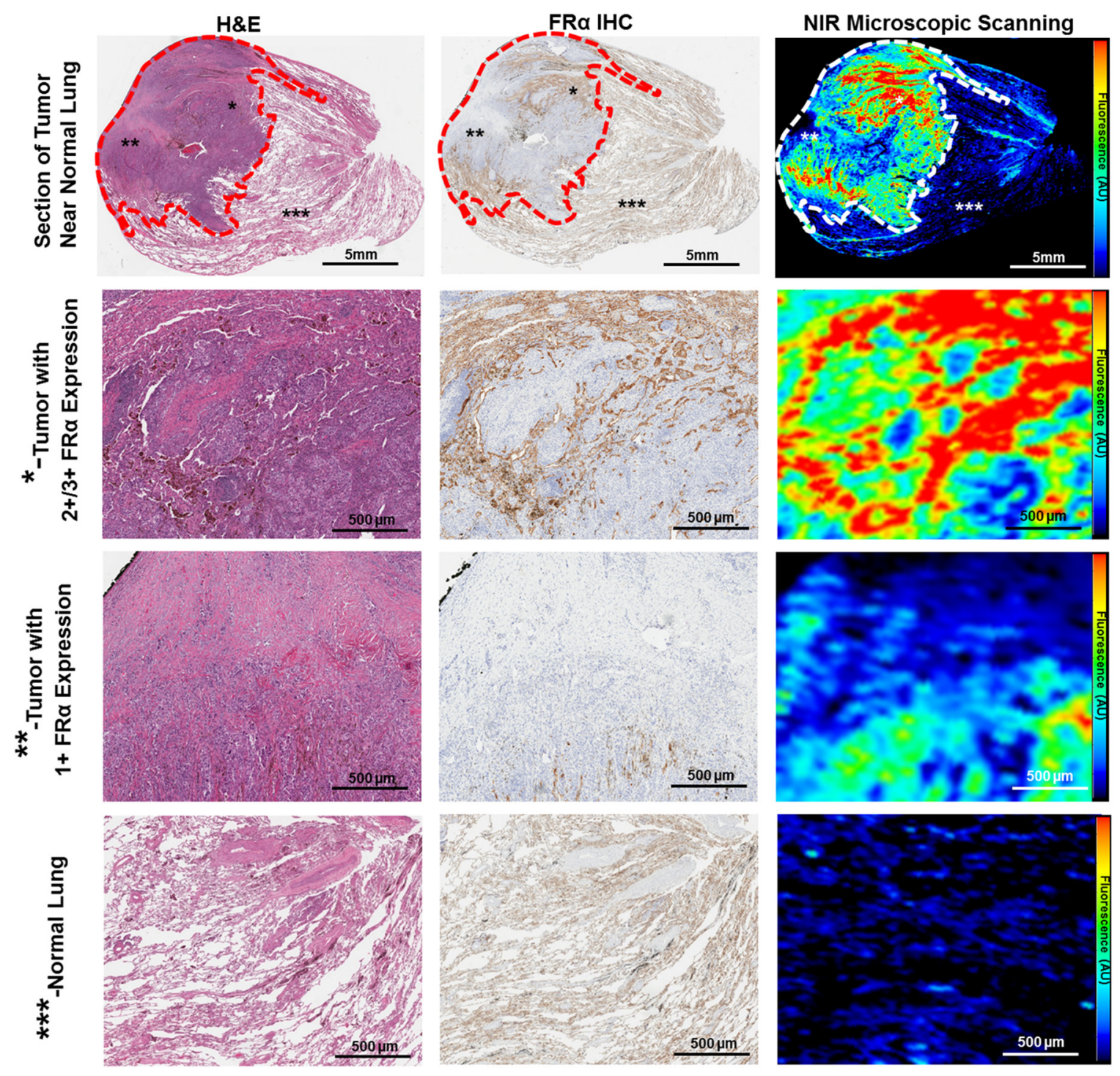

Figure 4: OTL38 accumulation within SCC is FRa-dependent. Each resected SCC underwent microscopic fluorescent scanning and correlative molecular analysis. Representative comprehensive analysis of resected SCC (Subject 9). Whole-section images were obtained and evaluated using H\&E staining, FR $\alpha$ IHC and a NIR microscopic scanning (top row). The tumor (gated in in dash marks) demonstrated strong fluorescence, particularly in areas of FR $\alpha$ expression. We noted increased fluorescence in areas of FR $\alpha$ overexpression $\left({ }^{*}\right.$ - second row), and moderate levels in those areas with $1+\mathrm{FR} \alpha$ staining $\left({ }^{* *}\right.$-third row). Normal pulmonary parenchyma displayed negligible fluorescence $\left({ }^{* * *}\right.$-bottom row). ${ }^{*}$ - Area of tumor with FR $\alpha$ overexpression $(2+/ 3+$ Staining $),{ }^{* *}$ - Area of tumor with low FR $\alpha$ expression $(1+$ or absent), ${ }^{* * *}$ - normal lung parenchyma. 
Table 3: Variables predicting in situ fluorescence

\begin{tabular}{lccc}
\hline Characteristics of fluorescent SCCs (n=9) & Coefficient & $\mathbf{9 5 \%}$ CI & p-value \\
\hline Depth of Nodule (cm) & -0.61 & -0.96 to -0.25 & $\mathbf{0 . 0 0 5}$ \\
Time to Imaging (hours) & 0.39 & -0.01 to 0.80 & 0.06 \\
Standard Uptake Value by PET & 0.29 & -0.08 to 0.23 & 0.29 \\
Nodule Location (RUL, RML, RLL, & 0.20 & -0.48 to 0.89 & 0.50 \\
LLL, vs LUL) & -0.15 & -1.59 to 1.28 & 0.80 \\
Nodule size (cm) & 0.71 & -1.15 to 2.57 & 0.81 \\
Amount of drug given (mg) & 0.02 & -0.18 to 0.23 & 0.81 \\
Age (years) & -0.06 & -1.77 to 1.64 & 0.93 \\
Gender (M vs F) & & \\
\hline
\end{tabular}

RUL-Right Upper Lobe; RML-Right Middle Low; RLL-Right Lower Lobe; LUL-Left Upper Lobe; LLL-Left Lower Lobe; M-Male; F-Female.
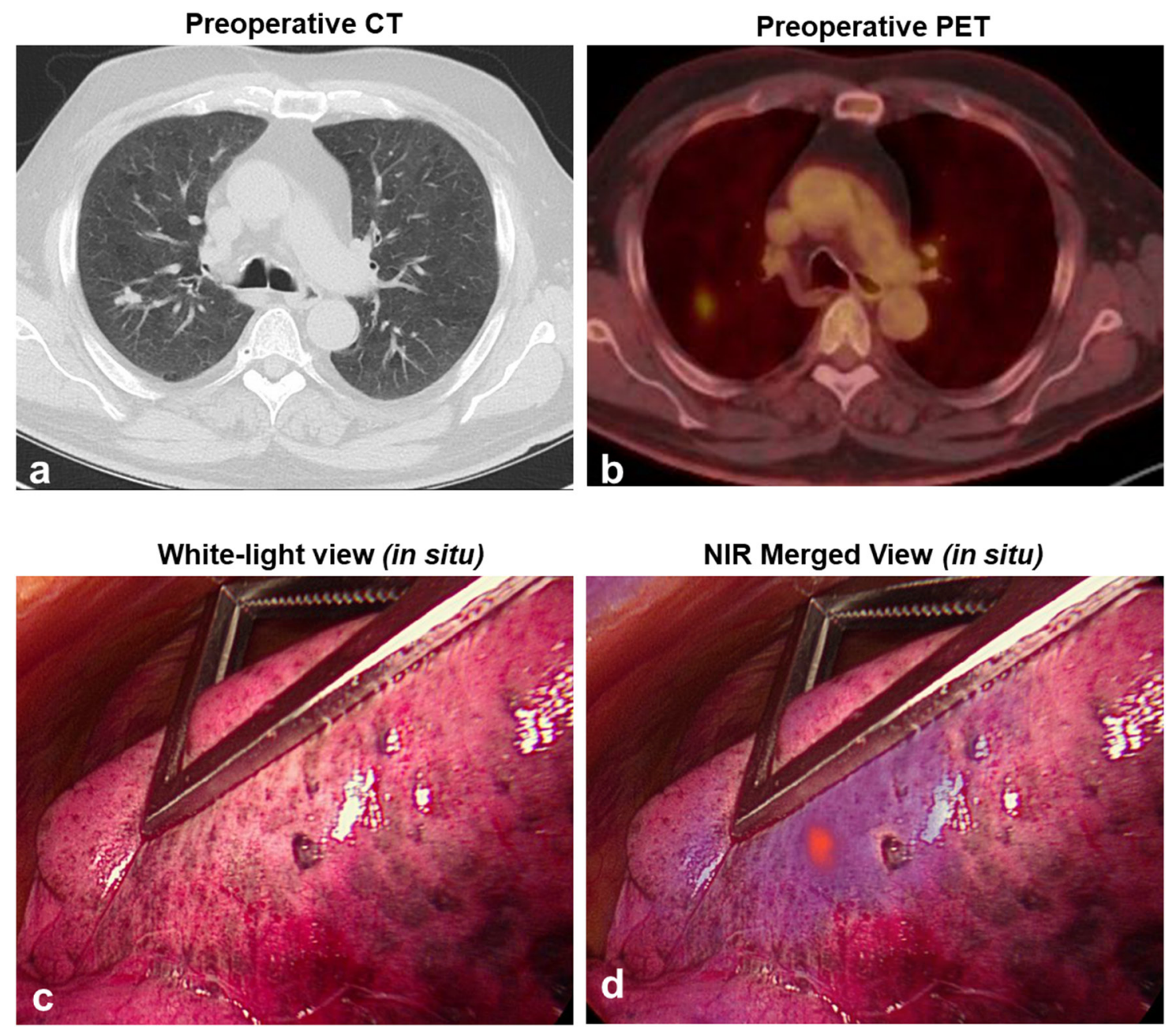

Figure 5: Representative example displaying FR-IMI localization of a small SCCs. Subject 9 presented with a metabolically active $1.1 \mathrm{~cm}$ nodule of the right upper lobe as determined by preoperative CT (a) and PET (b). Intraoperatively, the nodule could not be localized using standard visualization techniques (c); however, upon FR-IMI that nodule highly visible (d). 
IMI OTL38 which will mark the United States' first trial involving targeted IMI. These additional investigations will provide a better understanding of how FR-IMI can impact care for patients undergoing minimally invasive pulmonary resection for NSCLC.

\section{MATERIALS AND METHODS}

\section{Study design}

This clinical trial was approved by the University of Pennsylvania's Institutional Review Board. The primary objectives of this study were to determine if OTL38 accumulates within SCCs and to assess in vivo and ex vivo tumor fluorescence. Secondary objectives included the determination of fluorescent dependence on patient and tumor variables including FR $\alpha$ expression.

Twelve subjects provided informed consent and were enrolled between July 2015 and January 2017. Sample size and study design were based on consensus guidelines provided by the World Molecular Imaging Society [31]. All subjects presented with solitary pulmonary nodules that were histologically confirmed to be SCCs. All identified subjects underwent preoperative high-resolution CT scanning which was reviewed by a thoracic radiologist to confirm the presence pulmonary nodule and identify other suspicious nodules. Each subject also underwent preoperative ${ }^{18} \mathrm{FDG}$-positron emission tomography (PET). For imaging studies obtained outside the University of Pennsylvania Health System (UPHS), at least one UPHS thoracic radiologist reviewed preoperative imaging to confirm the presence of pulmonary nodules, rule-out synchronous disease, and confirm findings of ${ }^{18}$ FDG-positron emission tomography. Based on limitations in depth of penetration recognized in our prior experience with NIR imaging, we selected subjects SCCs within $4 \mathrm{~cm}$ of the visceral pleural surface on preoperative imaging [13]. A Transparent Reporting of Evaluations with Nonrandomized Designs (TREND) flow diagram with complete inclusion and exclusion criteria is provided (Supplementary Figure 1).

All subjects were scheduled for minimally invasive pulmonary resection (video-assisted thoracic surgery-VATS). In the preoperative holding area, study participants received $0.025 \mathrm{mg} / \mathrm{kg}$ of OTL38 by intravenous infusion 3-6 hours prior to resection as previously described [13]. During VATS, surgeons first utilized standard thoracoscopic visualization (white-light thoracoscopy) and finger palpation to identify known tumors. After identification of preoperatively identified nodules, molecular imaging was used to confirm lesion fluorescence. If the preoperatively identified nodule was unidentifiable by white-light thoracoscopy or finger palpation, localization using fluorescence guidance was attempted. If additional fluorescent lesions were identified, they were wedge resected. All specimens were then imaged ex vivo prior to submitting for histopathologic examination by a pulmonary pathologist.

\section{Study drug}

OTL38 $\left(\mathrm{C}_{61} \mathrm{H}_{63} \mathrm{~N}_{9} \mathrm{Na}_{4} \mathrm{O}_{17} \mathrm{~S}_{4}\right.$; molecular weight: $1414.42 \mathrm{Da}$ ) is composed of a folate analogue conjugated to an NIR fluorescent dye as previously described [3]. OTL38 maximally excites at a wavelength of 774-776 nm and has a peak emission of 794-796 nm [3, 9]. OTL38 ( $>96 \%$ purity) was obtained from On Target Laboratories (West Lafayette, IN, USA). All subjects received 0.025 $\mathrm{mg} / \mathrm{kg}$ of intravenous OTL38 through a peripheral vein 3 to 6 hours prior to resection. OTL38 dosage and time to imaging was based on previous human $[3,9]$ and canine pharmacokinetic data [32].

\section{Imaging device}

In situ, real-time fluorescent imaging was performed using an Iridium ${ }^{\circledR}$ system optimized for detection of OTL38 (Visionsense Corp, Philadelphia, PA) as previously described [3]. The Iridium $\AA$ is a high definition, dual band (white light and NIR) camera system capable of emitting and detection light in the NIR spectrum. An excitation source with a wavelength of $785 \mathrm{~nm}$ was utilized, and fluorescence was detected using a bandpass filter selective to light ranging from 800 to $835 \mathrm{~nm}$. During VATS, the Iridium ${ }^{\circledR}$ was equipped with a $5 \mathrm{~mm}$, 0 -degree thoracoscope. During in situ imaging during VATS, the distance was normalized using the Iridium ${ }^{\circledR}$ 's "distance lock" feature. For ex vivo back table evaluation of specimens, a free-standing exoscope was utilized. By exoscopic evaluation, imaging was performed at a distance of 15 inches. Of note, during both in situ and ex vivo imaging, the "autogain" function was utilized, and power was maintained at the default setting.

\section{Fluorescent and histopathologic assessment of specimen}

Excised specimens were formalin fixed and paraffin embedded. Sequential $5 \mu \mathrm{m}$ sections were obtained and underwent comprehensive histopathologic and fluorescent analysis by a board-certified thoracic pathologist. Sections were stained using standard hematoxylin/eosin (H\&E) staining. Immunohistochemical (IHC) staining for FR $\alpha$ was performed using a monoclonal antibody, 26B3.F2 (Morphotek Inc., PA) as previously described [13]. Once stained, a certified pulmonary pathologist manually scored specimen using an established scoring system ranging from 0 to $3+$ as previously described [13]. Briefly, a score of 0 corresponded with absence of staining; $1+$ equaled faint staining on luminal borders; $2+$ equaled moderate staining on apical and sometimes lateral borders and $3+$ indicated strong circumferential staining. The tumor was considered positive when more than $10 \%$ of malignant 
cells were positively stained. Lastly, in order to understand OTL38 accumulation patterns at a microscopic level, an additional unstained $5 \mu \mathrm{m}$ section was evaluated using a NIR microscopic scanner (Odyssey, LiCor, Lincoln, NE).

\section{Statistical analysis}

Post hoc image analysis was performed to quantify the amount of fluorescence using region of interest (ROI) software within ImageJ (free software program available through the National Institute of Health; http:// rsb.info.nih.gov/ij). A background fluorescence level was similarly obtained, and tumor-to-background (TBR) was calculated for all identified lesions. A TBR was utilized to control for extrinsic variables including ambient operating room signal, natural fluorophores, and systemic patient variables. Data are presented as median (IQR) unless otherwise noted. Given the small sample size, differences between two groups were compared by the Mann-Whitney test. Given the exploratory nature of this study, multiple prediction models were made using linear regression to assess patient and histopathologic variables that related to tumor fluorescence. All comparisons were made use Stata Statistical Software: Release 14 (College Station, TX: StataCorp LP). A p-value of 0.05 or less was considered statistically significant.

\section{Abbreviations}

AE: adverse event; BMP: basic metabolic panel; CBC: complete blood count; CT: computed tomography; CTCAE: Common Terminology Criteria for Adverse Events; FR $\alpha$ : folate receptor alpha; H\&E: hematoxylin and eosin; ICG: indocyanine green; IHC: immunohistochemistry; IMI: intraoperative molecular imaging; IQR: interquartile range; LFTs: liver function tests; $\mathrm{NaCl}$ : sodium chloride; NIR: near infrared; NSCLC: non: small cell lung cancer; PET: positron emission tomography; ROI: region of interest; SUV: standardized uptake value; TBR: tumor to background fluorescence ratio; VATS: video assisted thoracic surgery.

\section{Author contributions}

JDP, SS contributed to conceptualization, data curation, formal analysis, validation, manuscript preparation and review. AND, LX, CC, CC MS LFS participated in data curation, formal analysis, and manuscript review. LL, CD SN, SAK, PSL participated in formal analysis, manuscript preparation and manuscript review.

\section{ACKNOWLEDGMENTS}

The authors would like to thank On Target Laboratories for providing the study drug.

\section{CONFLICTS OF INTEREST}

SAK and PL are a Board Members of On Target Laboratories, producers of the study drug (OTL38).

\section{FUNDING}

JDP as supported by a grant from the American Philosophical Society, the NIH (1F32CA210409), and the Association for Academic Surgery Research Grant. SS was supported by the NIH (R01 CA193556).

\section{REFERENCES}

1. Predina JD, Keating J, Patel N, Nims S, Singhal S. Clinical implications of positive margins following non-small cell lung cancer surgery. J Surg Oncol. 2016; 113:264-9. https:// doi.org/10.1002/jso.24130.

2. Cerfolio RJ, Bryant AS. Is palpation of the nonresected pulmonary lobe(s) required for patients with non-small cell lung cancer? A prospective study. J Thorac Cardiovasc Surg. 2008; 135:261-8. https://doi.org/10.1016/j. jtcvs.2007.08.062.

3. Predina JD, Newton AD, Keating J, Barbosa EM Jr, Okusanya O, Xia L, Dunbar A, Connolly C, Baldassari MP, Mizelle J, Delikatny EJ, Kucharczuk JC, Deshpande C, et al. Intraoperative Molecular Imaging Combined With Positron Emission Tomography Improves Surgical Management of Peripheral Malignant Pulmonary Nodules. Ann Surg. 2017. https://doi.org/10.1097/SLA.0000000000002382.

4. Tipirneni KE, Warram JM, Moore LS, Prince AC, de Boer E, Jani AH, Wapnir IL, Liao JC, Bouvet M, Behnke NK, Hawn MT, Poultsides GA, Vahrmeijer AL, et al. Oncologic Procedures Amenable to Fluorescenceguided Surgery. Ann Surg. 2016. https://doi.org/10.1097/ SLA.0000000000002127.

5. Hoogstins CE, Tummers QR, Gaarenstroom KN, de Kroon CD, Trimbos JB, Bosse T, Smit VT, Vuyk J, van de Velde CJ, Cohen AF, Low PS, Burggraaf J, Vahrmeijer AL. A Novel Tumor-Specific Agent for Intraoperative Near-Infrared Fluorescence Imaging: A Translational Study in Healthy Volunteers and Patients with Ovarian Cancer. Clin Cancer Res. 2016; 22:2929-38. https://doi. org/10.1158/1078-0432.CCR-15-2640.

6. Lamberts LE, Koch M, de Jong JS, Adams ALL, Glatz J, Kranendonk MEG, Terwisscha van Scheltinga AGT, Jansen L, de Vries J, Lub-de Hooge MN, Schroder CP, JorritsmaSmit A, Linssen MD, et al. Tumor-Specific Uptake of Fluorescent Bevacizumab-IRDye800CW Microdosing in Patients with Primary Breast Cancer: A Phase I Feasibility Study. Clin Cancer Res. 2017; 23:2730-41. https://doi. org/10.1158/1078-0432.CCR-16-0437.

7. Madajewski B, Judy BF, Mouchli A, Kapoor V, Holt D, Wang MD, Nie S, Singhal S. Intraoperative near-infrared imaging of surgical wounds after tumor resections can 
detect residual disease. Clin Cancer Res. 2012; 18:5741-51. https://doi.org/10.1158/1078-0432.CCR-12-1188.

8. Rosenthal EL, Warram JM, de Boer E, Chung TK, Korb ML, Brandwein-Gensler M, Strong TV, Schmalbach CE, Morlandt AB, Agarwal G, Hartman YE, Carroll WR, Richman JS, et al. Safety and Tumor Specificity of Cetuximab-IRDye 800 for Surgical Navigation in Head and Neck Cancer. Clin Cancer Res. 2015; 21:3658-66. https:// doi.org/10.1158/1078-0432.CCR-14-3284.

9. van Dam GM, Themelis G, Crane LM, Harlaar NJ, Pleijhuis RG, Kelder W, Sarantopoulos A, de Jong JS, Arts HJ, van der Zee AG, Bart J, Low PS, Ntziachristos V. Intraoperative tumor-specific fluorescence imaging in ovarian cancer by folate receptor-alpha targeting: first in-human results. Nat Med. 2011; 17:1315-9. https://doi.org/10.1038/nm.2472.

10. Vahrmeijer AL, Hutteman M, van der Vorst JR, van de Velde CJ, Frangioni JV. Image-guided cancer surgery using near-infrared fluorescence. Nat Rev Clin Oncol. 2013; 10:507-18. https://doi.org/10.1038/nrclinonc.2013.123.

11. Keating JJ, Kennedy GT, Singhal S. Identification of a subcentimeter pulmonary adenocarcinoma using intraoperative near-infrared imaging during video-assisted thoracoscopic surgery. J Thorac Cardiovasc Surg. 2015; 149:e51-3. https://doi.org/10.1016/j.jtcvs.2014.10.081.

12. Predina JD, Keating J, Dunbar A, Connolly C, Singhal S. Near-Infrared Intraoperative Molecular Imaging Can Enhance Malignant Pleural Mesothelioma Resection. The 25th Annual Meeting of the Asian Society for Cardiovascular and Thoracic Surgery. (Seoul, Korea). (2017).

13. Predina JD, Gaughan C, Jarrar D, Pechet T, Kucharczuk J, Low P, Singhal S. (2017). A Phase I Clinical Trial of Targeted Intraoperative Molecular Imaging for Pulmonary Adenocarcinomas. STS 53rd Annual Meeting. (Houston, TX, USA).

14. Hachey KJ, Gilmore DM, Armstrong KW, Harris SE, Hornick JL, Colson YL, Wee JO. Safety and feasibility of near-infrared image-guided lymphatic mapping of regional lymph nodes in esophageal cancer. J Thorac Cardiovasc Surg. 2016; 152:546-54. https://doi.org/10.1016/j. jtcvs.2016.04.025.

15. Marbacher S, Klinger E, Schwyzer L, Fischer I, Nevzati E, Diepers M, Roelcke U, Fathi AR, Coluccia D, Fandino J. Use of fluorescence to guide resection or biopsy of primary brain tumors and brain metastases. Neurosurg Focus. 2014; 36:E10. https://doi.org/10.3171/2013.12.FOCUS13464.

16. Keating JJ, Nims S, Venegas O, Jiang J, Holt D, Kucharczuk JC, Deshpande C, Singhal S. Intraoperative imaging identifies thymoma margins following neoadjuvant chemotherapy. Oncotarget. 2016; 7:3059-67. https://doi. org/10.18632/oncotarget.6578.

17. Keating J, Newton A, Venegas O, Nims S, Zeh R, Predina J, Deshpande C, Kucharczuk J, Nie S, Delikatny EJ, Singhal S. Near-Infrared Intraoperative Molecular Imaging Can Locate Metastases to the Lung. Ann Thorac Surg. 2016. https://doi.org/10.1016/j.athoracsur.2016.08.079.
18. Kennedy GT, Okusanya OT, Keating JJ, Heitjan DF, Deshpande C, Litzky LA, Albelda SM, Drebin JA, Nie S, Low PS, Singhal S. The Optical Biopsy: A Novel Technique for Rapid Intraoperative Diagnosis of Primary Pulmonary Adenocarcinomas. Ann Surg. 2015; 262:602-9. https://doi. org/10.1097/SLA.0000000000001452.

19. Okusanya OT, DeJesus EM, Jiang JX, Judy RP, Venegas OG, Deshpande CG, Heitjan DF, Nie S, Low PS, Singhal $\mathrm{S}$. Intraoperative molecular imaging can identify lung adenocarcinomas during pulmonary resection. J Thorac Cardiovasc Surg. 2015; 150:28-35 e1. https://doi. org/10.1016/j.jtcvs.2015.05.014.

20. Cagle PT, Zhai QJ, Murphy L, Low PS. Folate receptor in adenocarcinoma and squamous cell carcinoma of the lung: potential target for folate-linked therapeutic agents. Arch Pathol Lab Med. 2013; 137:241-4. https://doi.org/10.5858/ arpa.2012-0176-OA.

21. O'Shannessy DJ, Yu G, Smale R, Fu YS, Singhal S, Thiel RP, Somers EB, Vachani A. Folate receptor alpha expression in lung cancer: diagnostic and prognostic significance. Oncotarget. 2012; 3:414-25. https://doi.org/10.18632/ oncotarget. 489.

22. Society AC. (2017). Non-Small Cell Lung Cancer.

23. Mahalingam SK, Roy J, Low P. (2013). Evaluation of pteroyl-amino acid-NIR dye conjugates for tumor targeted fluorescence guided surgery. 246th American Chemical Society National Meeting and Exposition. (Indianapolis, IN, USA).

24. Parker N, Turk MJ, Westrick E, Lewis JD, Low PS, Leamon CP. Folate receptor expression in carcinomas and normal tissues determined by a quantitative radioligand binding assay. Anal Biochem. 2005; 338:284-93. https://doi. org/10.1016/j.ab.2004.12.026.

25. De Jesus E, Keating JJ, Kularatne SA, Jiang J, Judy R, Predina J, Nie S, Low P, Singhal S. Comparison of Folate Receptor Targeted Optical Contrast Agents for Intraoperative Molecular Imaging. Int J Mol Imaging. 2015; 2015:469047. https://doi.org/10.1155/2015/469047.

26. Stoffels I, Morscher S, Helfrich I, Hillen U, Leyh J, Burton NC, Sardella TC, Claussen J, Poeppel TD, Bachmann HS, Roesch A, Griewank K, Schadendorf D, et al. Metastatic status of sentinel lymph nodes in melanoma determined noninvasively with multispectral optoacoustic imaging. Sci Transl Med. 2015; 7:317ra199. https://doi.org/10.1126/ scitranslmed.aad1278.

27. Schmidt F, Dittberner A, Koscielny S, Petersen I, GuntinasLichius O. Feasibility of real-time near-infrared indocyanine green fluorescence endoscopy for the evaluation of mucosal head and neck lesions. Head Neck. 2017; 39:234-40. https:// doi.org/10.1002/hed.24570.

28. Hachey KJ, Colson YL. Current innovations in sentinel lymph node mapping for the staging and treatment of resectable lung cancer. Semin Thorac Cardiovasc Surg. 2014; 26:201-9. https://doi.org/10.1053/j. semtcvs.2014.09.001. 
29. Keating J, Singhal S. Novel Methods of Intraoperative Localization and Margin Assessment of Pulmonary Nodules. Semin Thorac Cardiovasc Surg. 2016; 28:127-36. https://doi.org/10.1053/j.semtcvs.2016.01.006.

30. Rosenthal EL, Moore LS, Tipirneni K, de Boer E, Stevens TM, Hartman YE, Carroll WR, Zinn KR, Warram JM. Sensitivity and Specificity of Cetuximab-IRDye800CW to Identify Regional Metastatic Disease in Head and Neck Cancer. Clin Cancer Res. 2017. https://doi. org/10.1158/1078-0432.CCR-16-2968.

31. Tummers WS, Warram JM, Tipirneni KE, Fengler J, Jacobs P, Shankar L, Henderson L, Ballard B, Pogue BW, Weichert
JP, Bouvet M, Sorger J, Contag CH, et al. Regulatory Aspects of Optical Methods and Exogenous Targets for Cancer Detection. Cancer Res. 2017; 77:2197-206. https:// doi.org/10.1158/0008-5472.CAN-16-3217.

32. Keating JJ, Runge JJ, Singhal S, Nims S, Venegas O, Durham AC, Swain G, Nie S, Low PS, Holt DE. Intraoperative near-infrared fluorescence imaging targeting folate receptors identifies lung cancer in a large-animal model. Cancer. 2017; 123:1051-60. https://doi.org/10.1002/ cncr.30419.

33. SERVICES USDOHAH. Common Terminology Criteria for Adverse Events (CTCAE) Version 4.0. 2010. 